Ulrike Verch*

\title{
Freiwillig tätig, aber vertraglich gebunden: Rechtsbeziehungen bei ehrenamtlichen Tätigkeiten in Bibliotheken
}

https://doi.org/10.1515/bfp-2020-0113

Zusammenfassung: Ehrenamtliche Tätigkeiten in Bibliotheken bedürfen einer vertraglichen Regelung und werden im Regelfall als Auftragsverhältnis nach dem Bürgerlichen Gesetzbuch ausgestaltet. Der Beitrag erläutert die rechtlichen Rahmenbedingungen für das ehrenamtliche Engagement in Bibliotheken und setzt den Fokus auf die vertragliche Gestaltung der Tätigkeitsvereinbarung und stellt die Unterschiede zum Arbeitsvertrag dar.

Schlüsselwörter: Ehrenamt; Recht; Vertrag

\section{Voluntary but Contractually Bound: Legal Relationships in the Case of Voluntary Work in Libraries}

Abstract: Voluntary work in libraries requires a contractual arrangement and is usually structured as a contractual relationship of personal service in accordance with the German Civil Code. The article explains the legal framework for volunteer work in libraries and focuses on the contractual design of the service agreement - and shows the differences to the employment contract.

Keywords: Voluntary work; law; contract

\section{Einleitung}

Das Ehrenamt ist seit vielen Jahren in zahlreichen Öffentlichen und auch etlichen wissenschaftlichen Bibliotheken in Deutschland fest etabliert und eine wichtige Stütze der aktiven Bibliotheksarbeit in den Kommunen. ${ }^{1}$ Allein bei den Bücherhallen Hamburg engagieren sich neben den 420 Mitarbeiter*innen rund 600 Personen freiwillig in ge-

1 Das Ehrenamt zeichnet sich durch folgende Aspekte aus: Unentgeltlichkeit, Freiwilligkeit, Öffentlichkeit und Gemeinwohlorientierung. Mehr über den Begriff des Ehrenamtes nachzulesen bei Zeddies (2011a) 9 ff.

*Kontaktperson: Prof. Dr. Ulrike Verch, ulrike.verch@haw-hamburg.de meinnützigen Projekten u. a. zur Leseförderung, Integration und Seniorenarbeit. ${ }^{2}$ An der Stadtbibliothek Mühlheim an der Ruhr bringen freiwillig Tätige für sehbehinderte Personen eine lokale Hörzeitung heraus ${ }^{3}$ und betreiben eine antiquarische Schmökerstube. ${ }^{4}$ In anderen Orten werden komplette Schul ${ }^{5}$ oder Stadtteilbibliotheken ${ }^{6}$ ausschließlich mit der Hilfe von engagierten Bürger ${ }^{\star}$ innen betrieben und die kirchliche Büchereiarbeit ist traditionell ehrenamtlich geprägt: Über 90 \% der Einrichtungen werden durch freiwillige Helferinnen unterhalten. ${ }^{7}$

Auch wenn die inhaltliche Bandbreite und Vielfalt ehrenamtlicher Aufgaben, die Bürger*innen in ihrer Freizeit für Bibliotheken übernehmen können, sehr groß ist, ${ }^{8}$ unterliegen sie grundsätzlich vergleichbaren juristischen Rahmenbedingungen. Gleichwohl, ob bei der Seniorenarbeit oder bei Leseförderungsveranstaltungen mit Kleinkindern, entstehen sowohl für die Bibliotheken als auch für die freiwillig Tätigen jeweils Rechte und Pflichten, die durch vertragliche Vereinbarungen geregelt werden.

Auf welche Weise sich die Rechtsbeziehungen zwischen Ehrenamtlichen und Bibliotheken vertraglich gestalten lassen und welche gesetzlichen Rahmenbedingungen dabei zu beachten sind, wird im folgenden Beitrag näher dargestellt.

2 https://www.buecherhallen.de/ueber-uns.html.

3 https://www.muelheim-ruhr.de/cms/echo_muelheim_-_die_hoer zeitung3.html.

4 https://www.muelheim-ruhr.de/cms/schmoekerstube.html.

5 https://frankfurt.de/service-und-rathaus/verwaltung/aemter-un d-institutionen/stadtbuecherei/startseite-ueber-uns/ehrenamt.

6 https://www.vdb-online.org/veranstaltung/341/harre.pdf.

7 Zeddies (2011b) 1.

8 Siehe Übersicht bei Umlauf (2011) Kap. 4.7.7. 


\section{Ist ein Vertrag für bürgerschaftliches Engagement notwendig?}

Wenn der Umfang und die Dauer der ehrenamtlichen Tätigkeit nicht groß sind, könnte sich für manche Bibliothek die Frage stellen, ob es überhaupt eines Vertrages bedarf. Die Antwort hierauf ist eindeutig ja, denn nur so lässt sich das Ehrenamt verlässlich und verbindlich für beide Seiten regeln, auch ohne großen Aufwand. Ein Vertragsschluss bedeutet nicht unbedingt aufwendige und komplizierte Vertragsdokumente. Auch mittels mündlicher oder digitaler Absprachen werden Verträge geschlossen. Sobald zwei übereinstimmende Willenserklärungen vorliegen, die auf die Bewirkung einer Rechtsfolge gerichtet sind, kommt gemäß §§ $145 \mathrm{ff}$. Bürgerliches Gesetzbuch (BGB) ein zweiseitiger Vertrag zustande, egal auf welche Weise dieser geschlossen wurde. ${ }^{9}$

Ohne Vertragsschluss wäre die ehrenamtliche Tätigkeit, rechtlich betrachtet, ein bloßes, unverbindliches Gefälligkeitsverhältnis. Dann könnte die Bibliothek keinerlei Einfluss auf die Ausgestaltung der durchzuführenden Dienstleistungen nehmen, und die Freiwilligen wären hinsichtlich der Haftung und Kostenerstattung nicht privilegiert. Deshalb sind verbindliche Vertragsregelungen erforderlich, auch um das gewünschte Qualitätsniveau und die Kontinuität der ehrenamtlichen Arbeit sicherzustellen. ${ }^{10}$

Da sich im Streitfall mündliche Absprachen nicht nachlesen und nur schwer beweisen lassen, ist grundsätzlich eine Textform für die vertragliche Vereinbarung $\mathrm{zu}$ empfehlen. Wichtig ist eine gute Dokumentation des Vertragstextes und des Umstands, dass sich beide Vertragsparteien auf diesen Text verständigt haben. Dazu sind nicht unbedingt persönliche Unterschriften notwendig, ein archivierter und datierter Mailwechsel reicht in der Regel aus. ${ }^{11}$

Sollte die Bibliothek Musterverträge für die ehrenamtliche Tätigkeit einsetzen, sind zusätzliche Nebenabsprachen nicht ausgeschlossen und können im Einzelfall sinnvoll sein. Sie sollten am besten als Änderungen und Ergänzungen zum Vertragstext schriftlich festgehalten

9 Bürgerliches Gesetzbuch (BGB) in der Fassung vom 02.01.2002 (BGBl. S. 42), zuletzt geändert am 12.06.2020.

10 So auch Umlauf (2011) Kap. 4.7.8.

11 Weitere Informationen zur Formfreiheit von Verträgen sind auf den Webseiten der Bundeszentrale für politische Bildung nachzulesen: https://www.bpb.de/nachschlagen/lexika/recht-a-z/22188/form vorschriften. und ebenfalls gut dokumentiert werden. Vertragsänderungen oder Ergänzungen sind im beiderseitigen Einvernehmen mit Wirkung für die Zukunft nach § 311 BGB jederzeit möglich.

Bei der konkreten vertraglichen Ausgestaltung gilt nach deutschem Recht Vertragsfreiheit, so dass inhaltlich ein großer Gestaltungsspielraum besteht. ${ }^{12}$ Neben der Grenze der Sittenwidrigkeit gem. §138 BGB, die u.a. auch eine Ausbeutung im Arbeitsbereich zum Beispiel bei einem groben Missverhältnis zwischen Leistung und Gegenleistung, verbietet, ${ }^{13}$ ist $\mathrm{zu}$ beachten, dass die vereinbarten ehrenamtlichen Aufgaben nicht als Arbeitsvertrag ausgestaltet werden, da ansonsten das enge juristische Korsett des Arbeitsrechts greift. ${ }^{14}$ Selbst wenn der Vertrag nicht als Arbeitsvertrag bezeichnet wird, können die der Bibliothek gegenüber zu erbringenden Dienstleistungen nach ihrem wahren Geschäftsinhalt als zu vergütende Arbeitsleistungen angesehen werden, wenn sie wie in einem hauptamtlichen Arbeitsverhältnis ausgestaltet sind. Das wäre insbesondere dann der Fall, wenn die Bibliothek die Arbeitsbedingungen durch ein umfassendes Direktionsrecht einseitig gestaltet und zudem eine persönliche Abhängigkeit der ehrenamtlichen Person gegeben ist. In einem solchen Fall hätte diese die Möglichkeit, ihren Arbeitnehmerstatus gerichtlich einzuklagen. ${ }^{15}$ Deshalb ist es nicht nur für die Vertragsformulierungen, sondern auch bei der praktischen Umsetzung in der Bibliotheksarbeit wichtig, den Schwerpunkt der ehrenamtlichen Tätigkeit klar auf gemeinnützige Aufgaben im sozialen, pädagogischen oder karitativen Bereich zu legen.

\section{Ehrenamt im Auftragsverhältnis}

Im Gegensatz zur entgeltlichen Dienstleistung im Arbeitsverhältnis ist das Ehrenamt im BGB nicht als Standard-

12 Die Vertragsfreiheit ist aus dem Grundrecht der Allgemeinen Handlungsfreiheit nach Art. 2 Abs. 1 Grundgesetz abzuleiten.

13 Zur sittenwidrigen Lohnvergütung, siehe Bundesarbeitsgericht, Urteil vom 22.04.2009, Az. 5 AZR 436/08.

14 U.a. Kündigungsschutz, Sozialversicherungspflichten, Mindestlohn, Urlaubs- und Entgeltfortzahlungen, Mitbestimmung.

15 So erfolgreich geschehen bei einem Dienstverhältnis mit dem Bayerischen Landesamt für Denkmalpflege, siehe Bundesarbeitsgericht, Urteil vom 25.09.2013, Az. 10 AZR 282/12. In einem früheren Urteil zu ehrenamtlichen Tätigkeiten bei einer Telefonseelsorge hat das Bundesarbeitsgericht hingegen die Annahme eines Arbeitsverhältnisses abgelehnt, siehe Bundesarbeitsgericht, Urteil vom 29.08.2012, Az. 10 AZR 499/11. 
vertrag kodifiziert. Üblicherweise werden vertragliche Vereinbarungen für ehrenamtliche Tätigkeiten juristisch als Auftragsverhältnis nach $\S \S 662 \mathrm{ff}$. BGB bewertet. ${ }^{16}$ Kennzeichnend für einen Auftrag ist, dass der/die Beauftragte eine Dienstleistung freiwillig und unentgeltlich vornimmt, die ihm von der Bibliothek als Auftraggeber übertragen worden ist. Anders als beim Arbeitsvertrag kommt der Bibliothek kein umfassendes Direktionsrecht wie beim Arbeitsverhältnis zu, auch wenn der Auftraggeber in Bezug auf die vertraglich vereinbarte Dienstleistung ein begrenztes Weisungsrecht ausüben kann. ${ }^{17}$ Zudem kann der Vertrag gemäß § 671 BGB jederzeit von beiden Seiten mit sofortiger Wirkung gekündigt bzw. widerrufen werden. Allerdings ist der Beauftragte dabei verpflichtet, auf die Interessen der Auftraggebers insofern Rücksicht zu nehmen, als dass die Bibliothek die Möglichkeit haben muss, die übertragene Dienstleistung auf andere Weise vorzunehmen, es sei denn, es liegt ein wichtiger Kündigungsgrund vor. Darüber hinaus sind die freiwilligen Helfer^innen nach $\S 666$ BGB verpflichtet, wichtige Informationen an ihren Auftraggeber weiterzuleiten und auf Wunsch der Einrichtung Auskunft und Rechenschaft über ihre Tätigkeit zu geben.

Kosten für Aufwendungen, die im Rahmen des Auftrags entstanden sind, können sich die Ehrenamtlichen gemäß § 670 BGB von den Bibliotheken erstatten lassen, sofern diese Aufwendungen erforderlich waren und tatsächlich angefallen sind. Dies sind typischerweise Materialkosten, aber auch Gebühren, Fahrt-, Porto-, Handykosten oder sonstige notwendige Ausgaben können als Aufwandsentschädigung geltend gemacht werden. ${ }^{18}$

Manche Bibliotheken bieten darüber hinaus zusätzliche Leistungen wie zum Beispiel kostenlose Bibliotheksausweise, freier oder vergünstigter Eintritt bei Veranstaltungen, finanzierte Fortbildungen, Fachliteratur, Weihnachtsfeiern oder Neujahrsempfänge im Rathaus. ${ }^{19}$ Gesetzlich sind diese Leistungen jedoch nicht vorgeschrieben und müssen auch nicht in die Tätigkeitsvereinbarung aufgenommen werden, es sei denn, dass der Beauftragte diese Gratifikationen vertragsverbindlich regeln möchte. Zudem haben in den vergangenen Jahren die meisten Bundesländer die sog. Ehrenamtskarte eingeführt, mit der gemeinwohlorientierte Tätigkeiten der Ehrenamtlichen in

16 Schäfer (2020), in Münchener Kommentar zum BGB, § 662, Rn. 1. 17 Vgl. Landesarbeitsgericht Schleswig-Holstein, Urteil vom 23.11. 2016, Az. 3 Sa 214/16 [ECLI:DE:LARBGSH:2016:1123.3SA214.16.0A] zur Arbeitnehmereigenschaft eines ehrenamtlichen Rettungsassistenten. 18 Schäfer (2020), in Münchener Kommentar zum BGB, § 670, Rn. 9. 19 Vgl. Kulzer (2015) 196.
Form von Vergünstigungen und Rabatten honoriert werden sollen. ${ }^{20}$

Der von der Bibliothek verwendete Vertragsname spielt für die rechtliche Bewertung des Vertragsverhältnisses nur eine ungeordnete Rolle. Maßgeblich sind stets der objektiv vereinbarte Geschäftsinhalt und die konkrete Ausgestaltung der Dienstleistungen in der Praxis. Dabei sind alle Umstände des Einzelfalls in Betracht zu ziehen und in ihrer Gesamtheit zu würdigen. ${ }^{21}$ Als mögliche Benennungen bieten sich „Vereinbarung für Ehrenamtliche, „Ehrenamtsvereinbarung“, Ehrenamtsvertrag“, „Einsatzvereinbarung“, „Tätigkeitsvereinbarung“ oder auch nur "Vereinbarung" an, aber auch andere Titulierungen sind möglich und denkbar, solange das Wort Arbeitsvertrag gemieden wird. ${ }^{22}$

\begin{tabular}{|l|l|}
\hline Arbeitsvertrag mit Hauptamtlichen & Tätigkeitsvereinbarung mit Ehrenamtlichen \\
\hline Arbeitsvertrag nach § 611a BGB & Auftrag nach § 622 BGB \\
\hline Entgelt-Vergütung & Aufwandsentschädigung möglich \\
\hline persönliche Abhängigkeit & Tätigkeit erfolgt ehrenhalber \\
\hline umfassendes Direktionsrecht & Konsensprinzip \\
\hline Arbeitgeber legt Arbeitszeiten fest & Einsatzzeiten frei gestaltbar \\
\hline Kündigungsschutz & jederzeit beidseitig kündbar \\
\hline Mitbestimmung über Personalvertretung & keine Mitbestimmung \\
\hline Sozialversicherungsleistungen & nur Haftpflicht- und Unfallversicherung \\
\hline
\end{tabular}

Abb. 1: Unterschiede zwischen einem Arbeitsvertrag und einer Tätigkeitsvereinbarung

\section{Gibt es juristische Unterschiede bei verschiedenen Bezeichnungen für das Ehrenamt?}

Werden die ehrenamtlichen Tätigkeiten im Rahmen des Bundes- oder Jugendfreiwilligendienstes absolviert, gelten besondere gesetzliche Regelungen. So ist im Gesetz über den Bundesfreiwilligendienst (Bundesfreiwilligendienstgesetz - BFDG) ${ }^{23}$ genau geregelt, welche Rechte und Pflich-

20 Im Detail gibt es Unterschiede zwischen den einzelnen Bundesländern, hier das Beispiel aus Bayern: https://www.lbe.bayern.de/en gagement-anerkennen/ehrenamtskarte/.

21 Vgl. Bundesarbeitsgericht, Urteil vom 09.04.2014, Az. 10 AZR 590/13.

22 Siehe als Beispiele https://www.bz-niedersachsen.de/files/bznc3/content/PDF-Dateien/Basiskurs.Frewilligenarbeit.Mustervertrag.p df, https://www.bibliotheksverband.de/fileadmin/user_upload/DB V/themen/ehrenamt/Bayern_Vereinbarung_Muster_Ehrenamtliche.p df oder https://www.schulministerium.nrw.de/sites/default/files/do cuments/Einsatzvereinbarung_Ehrenamtliche.pdf.

23 Bundesfreiwilligendienstgesetz vom 28.04.2011 (BGBl. I S. 687), zuletzt geändert am 12.12.2019. 
ten im Rahmen des Freiwilligendienstes zu beachten sind, und gemäß § 9 BFDG, wie die vertragliche Vereinbarung konkret auszugestalten ist. Die Bibliothek kann dabei den Vertrag und die zu verpflichtende Person nur vorschlagen: Der Vertrag selbst wird zwischen der freiwilligen Person und der Bundesrepublik Deutschland geschlossen, die auch die Finanzierung trägt.

Ähnlich verhält es sich bei den Jugendfreiwilligendiensten. Sowohl das Freiwillige Soziale Jahr (FSJ) als auch das Freiwillige Ökologische Jahr (FÖJ) sowie der Internationale Jugendfreiwilligendienst (IJFD) werden in einem eigenen Bundegesetz, dem Gesetz zur Förderung von Jugendfreiwilligendiensten (Jugendfreiwilligendienstegesetz - JFDG ${ }^{24}$ ausführlich geregelt. Nach $\S 11$ JFDG wird die Tätigkeitsvereinbarung zwischen der/dem Jugendlichen und dem Träger der Einsatzstelle geschlossen. Bei Öffentlichen Bibliotheken wären dies in der Regel die Kommunen als Gebietskörperschaft. Weitere ausführliche Informationen, auch bei rechtlichen Fragestellungen zu den Freiwilligendiensten, die durch den Bund gefördert werden, sind auf den Internetseiten des Bundesministeriums für Familie, Senioren, Frauen und Jugend sowie des Bundesamtes für Familie und zivilgesellschaftliche Aufgaben zu finden. ${ }^{25}$

Praktika werden in der Regel nicht als ehrenamtliche Tätigkeit angesehen, auch wenn sie unbezahlt sind. Sie dienen keinen gemeinnützigen Zwecken, sondern der Ausbildung und Berufsorientierung. Zudem gelten Sonderregelungen, wenn die Praktika im Rahmen einer Schuloder Hochschulausbildung verpflichtend vorgeschrieben sind. So sind beispielsweise für die Ausgestaltung der Praktikumstätigkeiten für Studierende der Hochschule für Angewandte Wissenschaften Hamburg im Studiengang Bibliotheks- und Informationsmanagement die aktuelle Studien- und Prüfungsordnung sowie die Praktikumsrichtlinien mit konkreten Vorgaben über Inhalte, Arbeitszeiten, Freistellungen oder sonstige organisatorische Fragestellungen maßgeblich. ${ }^{26}$

Abgesehen von diesen gesetzlichen Sonderformen spielt im Übrigen die Bezeichnung des Ehrenamtes für die juristische Bewertung keine Rolle. Ob die Bibliothek von Zeitspende, bürgerlichem Engagement, zivilgesellschaftli-

24 Jugendfreiwilligendienstegesetz vom 16.05.2008 (BGBl. I S. 842), zuletzt geändert am 12.12.2019 (BGBl. I S. 2652).

25 Siehe https://www.bmfsfj.de/bmfsfj/themen/engagement-undgesellschaft/freiwilligendienste und https://www.bundesfreiwilligen dienst.de/service/downloads.html.

26 Siehe https://www.haw-hamburg.de/hochschule/design-medie n-und-information/departments/information/studium/praxisphase/ praktikum-bim/. chen Aufgaben oder gemeinwohlorientierten Einsätzen spricht, ist irrelevant. Maßgeblich ist nur, wie das Vertragsverhältnis konkret ausgestaltet ist, insbesondere bezüglich der Frage des Entgelts. Geringfügig Beschäftigte, Langzeitarbeitslose mit einem Ein-Euro-Job oder studentische Hilfskräfte arbeiten nicht im Ehrenamt, sondern haben einen Arbeitsvertrag. ${ }^{27}$ Auch die Abgrenzung zum Werkvertrag gemäß § 631 BGB ist zu beachten: Wenn einmalige Hilfstätigkeiten für die Bibliothek, wie beispielsweise die Reparatur eines Rechners oder die Gestaltung einer Weihnachtspostkarte, entlohnt werden, ist rechtlich von einem Werkvertrag auszugehen, auch wenn der gezahlte Geldbetrag nur gering ist. ${ }^{28}$

\section{Wer sind die Vertragsparteien bei einer Tätigkeitsvereinbarung?}

Hinsichtlich des Bürgers, der sich für die Bibliothek engagieren möchte, ist diese Frage leicht zu beantworten, da er als natürliche Person rechts ${ }^{29}$ - und geschäftsfähig ${ }^{30}$ ist. Nur bei Minderjährigen bedürfen Verträge, die nicht lediglich rechtlich vorteilhaft sind, der Zustimmung der Erziehungsberechtigten gemäß 107 BGB.

Auf Seiten der Bibliothek ist die Frage nach der Vertragspartei indes schwieriger zu beantworten, da die wenigsten Einrichtungen eigene Rechtspersönlichkeiten sind. Bei Öffentlichen Bibliotheken in kommunaler Trägerschaft werden die Verträge meist im Namen der Kommunen geschlossen, die als Gebietskörperschaften eigenständige juristische Personen und die Träger der Bibliotheken sind. Sie können privatrechtliche Verträge mit Wirkung für und gegen die Körperschaft entweder durch gesetzlich oder rechtsgeschäftlich bevollmächtigte Vertreter vornehmen. ${ }^{31}$

Bei privatrechtlichen Einrichtungen wie z.B. den Hamburger Bücherhallen in der Rechtsform einer Stiftung privaten Rechts ${ }^{32}$ ist die Institution eine selbständige juris-

27 Zur strikten Trennung von geringfügigen Beschäftigungsverhältnissen und bürgerschaftlichem Engagement, siehe Umlauf (2011) Kap. 4.7.11.

28 Bei einem Werkvertrag verpflichtet sich der Auftragnehmer gegen Zahlung durch den Besteller ein einmaliges Werk innerhalb eines gewissen Zeitrahmens herzustellen. Wiederkehrende Leistungen, die sich über einen längeren Zeitraum erstrecken, werden dagegen nicht werksvertraglich geregelt.

29 Nach $\S 1$ BGB.

30 Nach $\S 104$ ff BGB.

31 Neumayer (2001) 250.

32 https://www.buecherhallen.de/ueber-uns.html. 
tischer Person gemäß $\S 80$ BGB und kann damit im eigenen Namen Verträge schließen. ${ }^{33}$ Auch Vereine sind in der Regel gemäß § 21 und § 22 BGB entweder durch Eintragung im Vereinsregister oder durch staatliche Verleihung juristische Personen, so dass zahlreiche Bibliotheken ihre Förder- und Freundschaftsvereine nutzen, um Verträge mit den Ehrenamtlichen zu schließen. Neben der Ersparnis des Organisationsaufwands ist ein weiterer juristischer Vorteil, dass damit die Gefahr gemindert wird, dass sich Ehrenamtliche in ein Beschäftigungsverhältnis mit der Kommune einklagen können, da die vertraglichen Hauptleistungen nicht gegenüber der Bibliothek, sondern gegenüber dem Förderverein erbracht werden. Nachteilig an dieser Vertragskonstruktion ist hingegen, dass die Bibliotheken selbst keine Berichts- und Rechenschaftspflichten verlangen dürfen und zur Erfüllung datenschutzrechtlicher Anforderungen ggf. noch zusätzliche Vereinbarungen zur Auftragsverarbeitung mit dem Förderverein treffen müssen, wenn im Rahmen der ehrenamtlichen Tätigkeiten personenbezogene Nutzungsdaten bearbeitet werden. ${ }^{34}$

Möglich ist beim Förderverein auch das Modell, das alle Ehrenamtlichen Mitglied im Förderverein werden müssen und ihr ehrenamtlicher Einsatz in der Bibliothek über die Vereinssatzung geregelt ist. Diese Variante ist aber nicht zu empfehlen, da die Bibliothek keinen Einfluss auf die Vereinssatzung nehmen kann und auf die Ehrenamtlichen mit der Vereinsmitgliedschaft zusätzliche Pflichten zukommen.

Bei wissenschaftlichen Bibliotheken, in denen ehrenamtliche Tätigkeiten meist im Rahmen von historischen Erschließungsprojekten nur relativ selten durchgeführt werden, sind in der Regel die Hochschulen als Körperschaften öffentlichen Rechts die Vertragspartner, sofern keine Förder- und Freundesvereine diese Aufgabe übernehmen. ${ }^{35}$

Bei Katholischen Öffentlichen Bibliotheken, in denen die ehrenamtliche Büchereiarbeit weit verbreitet ist, sind schriftliche Tätigkeitsvereinbarungen mit Ehrenamtlichen eher unüblich, ${ }^{36}$ obgleich Religionsgemeinschaften als anerkannte Körperschaften des öffentlichen Rechts Verträge

33 Die Bücherhallen Hamburg haben für ihre Ehrenamtsprojekte eine eigene Tochtergesellschaft in Form einer gemeinnützigen Gesellschaft mit beschränkter Haftung gegründet, die Bücherhallen Medienprojekte $\mathrm{gGmbH}$, die für ihre Projekte die Tätigkeitsvereinbarungen mit den Ehrenamtlichen schließt.

$34 \mathrm{Zu}$ den rechtlichen Vor- und Nachteilen der Organisation des Ehrenamts über einen Förderverein siehe auch Gödan (1999) 988.

$35 \mathrm{Zu}$ den ehrenamtlichen Tätigkeiten in wissenschaftlichen Bibliotheken, siehe Umlauf (2011) Kap. 4.7.7.

36 Zeddies (2011b) 2. im eigenen Namen schließen können. ${ }^{37}$ Jedoch bieten die zwei Fachstellen, der Borromäusverein ${ }^{38}$ und der St. Michaelsbund für Bayern ${ }^{39}$, umfassende Unterstützungsangebote für Ehrenamtliche in Katholischen Öffentlichen Büchereien insbesondere in Form von Schulungen, Fortbildungen und Beratung an. ${ }^{40}$

Bei den evangelischen öffentlichen Büchereien engagiert sich das „Evangelische Literaturportal - Verband für Büchereiarbeit und Leseförderung “41 für die ehrenamtliche Arbeit, für die es umfassende Leitlinien entwickelt hat, in denen verbindliche Tätigkeitsvereinbarungen im Form eines Auftragsverhältnisses empfohlen werden..$^{42}$ Allerdings haben viele evangelische Landeskirchen eigene Richtlinien für die Freiwilligenarbeit verabschiedet, die bei der evangelischen Büchereiarbeit ebenfalls Anwendung finden. ${ }^{43}$

\section{Welche Inhalte sollte die Tätigkeitsvereinbarung umfassen?}

Grundsätzlich ist zu empfehlen, den Vertragstext nicht zu lang und $\mathrm{zu}$ ausführlich $\mathrm{zu}$ gestalten, da ansonsten die Gefahr besteht, dass zu lange Texte in vollem Umfang nicht gelesen werden, oder bei Detailregelungen schnell so kompliziert werden, dass das Textverständnis leidet und der Blick auf die Kernelemente des Vertrages verloren geht.

In jedem Fall sollte der Vertrag folgende Inhalte enthalten: ${ }^{44}$

\section{§ 1 Auftragsinhalt}

Folgende Tätigkeiten sind im Rahmen des Auftragsverhältnisses zu erfüllen:

37 https://www.bmi.bund.de/DE/themen/heimat-integration/staatund-religion/koerperschaftsstatus/koerperschaftsstatus-node.html. $38 \mathrm{https}$ //www.borromaeusverein.de/buechereiarbeit/.

39 https://www.st-michaelsbund.de/buechereien.html. 40 Zeddies (2011b) 2.

$41 \mathrm{https}$ //www.eliport.de/start/.

42 https://www.büchereiservice.de/fileadmin/redakteur/B\%C3\%B Ccherei_Service/Dokumente/Ehrenamt/Leitlinien_zum_Ehrenamt 13.pdf.

43 https://www.büchereiservice.de/ehrenamt/.

44 Angelehnt an den Mustervertrag von Gödan (1999). Weitere Beispiele für Musterverträge finden sich im Downloadbereich des Deutschen Bibliotheksverbandes unter https://www.bibliotheksverband. $\mathrm{de} / \mathrm{dbv} /$ themen/ehrenamt-in-bibliotheken/handbuch-fuer-das-ehre namts-management.html. 


\section{§ 2 Unentgeltlichkeit}

Die Tätigkeit der/des Beauftragten erfolgt ehrenhalber und unentgeltlich.

\section{§ 3 Weisungsrecht}

Der/die Beauftragte hat bei der Erfüllung seiner/ihrer Tätigkeiten den Weisungen derjenigen Person zu folgen, die hierzu vom Auftraggeber ermächtigt worden ist.

\section{§ 4 Dienstzeiten}

Die/der Beauftragte legt in Absprache mit der/dem Weisungsberechtigten ihre/seine Dienstzeiten fest.

\section{§ 5 Informationspflichten}

Im Falle der Verhinderung hat der/die Beauftragte den Auftraggeber zeitnah zu informieren.

\section{§ 6 Geheimnisschutz}

Es ist Stillschweigen über vertrauliche Informationen, auch nach Beendigung des Auftragsverhältnisses, zu wahren.

\section{§ 7 Aufwandsentschädigung}

Der Auftraggeber ersetzt der/dem Beauftragten alle Aufwendungen und Kosten, die zum Zwecke der Ausführung des Auftrags erforderlich sind. (Hier zählen insbesondere.....)

\section{§ 8 Vertragsauflösung}

Der Vertrag ist jederzeit beidseitig kündbar.

\section{§ 9 Geltung des Auftragsrechts}

Für diese Vereinbarung gelten die Bestimmungen des Auftragsrechts nach $\S \S 662-676$ BGB.

Darüber hinausgehende Vereinbarungen sind im Sinne der Vertragsfreiheit mit beiderseitigem Einverständnis jederzeit möglich. Aber nicht alle Fragestellungen rund um das Ehrenamt müssen vertraglich festgelegt werden, da viele Inhalte und Aspekte bereits durch gesetzliche Vorschriften allgemeingültig geregelt sind. ${ }^{45}$ So kommt beispielsweise dem Thema Haftung und Versicherung im Ehrenamt zwar eine große Bedeutung zu, um beide Vertragspartner hinreichend abzusichern, dies braucht aber nicht vertraglich gestaltet $\mathrm{zu}$ werden. Für bürgerschaftliches Engagement im Ehrenamt greift stets die gesetzliche Unfallversicherung nach $\S 2$ Abs. 1 Nr. 10 Buchstabe a So-

45 So zum Beispiel auch die Beachtung von Haus- und Benutzungsordnungen. zialgesetzbuch VII ${ }^{46}$ und die Haftpflicht für gemeinnützige Tätigkeiten wird im Schadensfall staatlich getragen. ${ }^{47}$ Deshalb bedürfen versicherungsrechtliche Inhalte in der Regel keiner gesonderten vertraglichen Vereinbarung, sondern können auch als reine Information über eine Webseite oder ein Infoblatt an die Ehrenamtlichen weitergereicht werden. Dasselbe gilt für das Steuerrecht, das sich nicht vertraglich gestalten lässt. Für Aufwandsentschädigungen, die kommunale Bibliotheken für tatsächlich angefallene Kosten im Auftragsverhältnis geleistet haben, muss grundsätzlich keine Einkommenssteuer gezahlt werden..$^{48}$ Zusätzliche Honorarleistungen können hingegen auch beim Ehrenamt einkommenssteuerpflichtig sein, fallen aber meistens nach $\S 3$ Nr. 26a Einkommenssteuergesetz ${ }^{49}$ unter den persönlichen Steuerfreibeitrag in Höhe von $720 € \mathrm{im} \mathrm{Jahr,} \mathrm{der} \mathrm{sog.} \mathrm{Ehrenamtspauschale.}{ }^{50}$

\section{Verpflichtung zum Daten- und Geheimnisschutz}

Neben der Tätigkeitsvereinbarung bedarf es vor Beginn der ehrenamtlichen Tätigkeit einer datenschutzrechtlichen Regelung. Da die Freiwilligen während ihres Engagements für Bibliotheken im Regelfall auch personenbezogene Daten verarbeiten oder Zugang zu Nutzerdaten haben, ist seitens der Einrichtung sicherzustellen, dass auch die Ehrenamtlichen über den korrekten Umgang mit personenbezogenen Daten Bescheid wissen und insbesondere unbefugte Datenweitergaben unterbleiben. Nach der Datenschutzgrundverordnung (DSGVO) müssen Ehrenamtliche, die als sog. unterstellte Personen nach Art. 29 DSGVO $^{51}$ anzusehen sind, über ihrer Pflichten unterrichtet und zur Vertraulichkeit sowie zur Einhaltung datenschutz-

46 Sozialgesetzbuch VII (Gesetzliche Unfallversicherung) vom 07.08.1996 (BGBl. I S. 1254), zuletzt geändert am 19.06.2020.

47 Versichert engagiert. Versicherungsschutz im Ehrenamt: https:// www.voev.de/wp-content/uploads/2018/05/Versicherungsschutz-i m-Ehrenamt_2018.pdf.

48 Vgl. Gödan (1999) 997.

49 Einkommensteuergesetz in der Fassung vom 8.10.2009 (BGBl. I S. 3366), zuletzt geändert am 12.08. 2020.

50 https://www.vereinswelt.de/ehrenamtspauschale.

51 Verordnung (EU) 2016/679 des Europäischen Parlaments und des Rates vom 27.04.2016 zum Schutz natürlicher Personen bei der Verarbeitung personenbezogener Daten, zum freien Datenverkehr und zur Aufhebung der Richtlinie 95/46/EG (Datenschutz-Grundverordnung), ABl. L 119 vom 04.05.2016, 1-88 [ELI: http://data.europa.eu/ eli/reg/2016/679/oj]. 
rechtlicher Grundsätze belehrt werden. ${ }^{52}$ Die deutsche Datenschutzkonferenz hat im Jahr 2018 einen Mustertext über die „Verpflichtung zur Vertraulichkeit und zur Einhaltung der datenschutzrechtlichen Anforderungen der DSGVO“ veröffentlicht, der für die ehrenamtliche Tätigkeiten in Bibliotheken zu empfehlen ist..$^{53}$ Der Text sollte sowohl von der verantwortlichen Stelle als auch von der verpflichteten Person datiert unterschrieben und anschließend dokumentiert werden. Wichtig zu beachten, dass Ehrenamtliche personenbezogene Daten nur auf Weisung der verantwortlichen Bibliothek verarbeiten dürfen und dass die Verpflichtung, personenbezogene Daten nicht unbefugt zu verarbeiten, auch nach Beendigung der Tätigkeit weitergilt.

Nimmt die ehrenamtliche Person während ihrer Tätigkeit Aufgaben wahr, die üblicherweise durch kommunale Amtsträger geleistet werden, kommt auch eine zusätzliche offizielle Verpflichtung nach dem Verpflichtungsgeset ${ }^{54}$ in Betracht, um eine Gleichstellung bei der strafrechtlichen Haftung sicherzustellen..$^{55}$ Inhalt der Verpflichtungserklärung sind vorrangig die Gewährleistung des Geheimnisschutzes sowie der Ausschluss der Bestechlichkeit und Vorteilsannahme. Die Ehrenamtlichen sind zunächst mündlich über ihre Pflichten und strafrechtliche Haftung zu belehren, um im Anschluss eine schriftliche Verpflichtungserklärung datiert zu unterschreiben. ${ }^{56}$

Des Weiteren ist vor Dienstantritt neben dem Nachweis der Personalien auch die Abgabe eines Führungszeugnisses nach $\S 30$ Abs. 5 Bundezentralregistergesetz ${ }^{57}$ erforderlich.

52 Die Belehrungspflicht ist aus Art. 32 Abs. 4 und Art. 5 Abs. 1 lit. f DSGVO abzuleiten. Eine Verpflichtung zur Einhaltung des Datengeheimnisses ist seit Inkrafttreten der DSGVO nach herrschender Meinung indes nicht mehr notwendig, vgl. Zilkens Rn. 150 (S. 92). 53 Der Mustertext ist u.a. auf der Webseite des Unabhängigen Landeszentrums für Datenschutz Schleswig-Holstein publiziert: https:// www.datenschutzzentrum.de/artikel/1235-Kurzpapier-Nr.-19-Unterri chtung-und-Verpflichtung-von-Beschaeftigten-auf-Beachtung-der-da tenschutzrechtlichen-Anforderungen-nach-der-DSGVO.html.

54 Verpflichtungsgesetz vom 02.03.1974 (BGBl. I S. 469), zuletzt geändert am 15.08.1974.

55 Vgl. Zilkens (2019) Rn. 152ff.

56 Ein guter Mustertext für eine entsprechende Verpflichtungserklärung findet sich auf der Homepage des Landratsamts Starnberg: https://www.lk-starnberg.de/media/custom/613_25671_1.PDF?14190 06570.

57 Bundeszentralregistergesetz in der Fassung vom 21.09.1984 (BGBl. I S. 1229), zuletzt geändert am 10.07.2020.

\section{Fazit und Zusammenfassung}

Auch wer nur freiwillig für gemeinnützige Zwecke in Bibliotheken tätig ist, bewegt sich nicht in einem rechtsfreien Raum. Viele Fragestellungen zu den konkreten Tätigkeitsaufgaben, Berichtspflichten, Weisungsrecht, Geheimnisschutz und Aufwandsentschädigung bedürfen einer verbindlichen Regelung und lassen sich am besten vertraglich gestalten. Für eine solche Tätigkeitsvereinbarung, die rechtlich als unentgeltliches Auftragsverhältnis anzusehen ist, gelten die Regelungen des Bürgerlichen Gesetzbuches, die den zwei Vertragsparteien sowohl in Hinsicht auf die Form des Vertragsschlusses als auch im Hinblick auf den Inhalt einen wesentlichen größeren Gestaltungsspielraum gewähren als im Arbeitsrecht. Insbesondere bei der Festlegung der Dienstzeiten, die nicht dem Weisungsrecht des Auftraggebers unterliegen, sind sehr flexible und individuelle Lösungen möglich. Um diese Offenheit und Flexibilität im Auftragsverhältnis mit den Ehrenamtlichen zu wahren, ist von einer starren Vertragsgestaltung mit umfangreichen Aufgabenbeschreibungen und $\mathrm{zu}$ detaillierten Einzelfallregelungen unbedingt abzuraten. Im Bedarfsfall lässt sich der Vertrag nachträglich stets noch einvernehmlich ändern oder ergänzen; und auch eine einseitige Kündigung bzw. Widerruf ist jederzeit möglich.

Und auch wenn die Tätigkeitsvereinbarung eine wichtige rechtliche Grundlage für das Ehrenamt darstellt, hängt die erfolgreiche Ausgestaltung des Ehrenamts nicht so sehr vom Vertrag als vielmehr von anderen Faktoren wie hoher Wertschätzung, regelmäßiger Kommunikation, guter Organisation, Beteiligung an Besprechungen und Gesprächskreisen, Fortbildungsmöglichkeiten und einer engagierten Betreuung ab. ${ }^{58}$ Diese Aspekte bedürfen keiner vertraglichen Regelung, sondern müssen vielmehr in der Praxis umgesetzt und gelebt werden. Und auch als Informationsmittel, um die Ehrenamtlichen über ihre Rechte und Pflichten bei der Tätigkeitsausübung in der Bibliothek zu informieren, ist ein juristischer Vertragstext nicht konzipiert und geeignet, zumal viele wichtige Fragestellungen beispielsweise zum Datenschutz, Urheberrecht oder zur Bibliotheksbenutzungsordnung in der Tätigkeitsvereinbarung auch gar nicht enthalten sind. Mit gut verständlichen und attraktiv gestalteten Informationsbroschüren und/ oder Internetseiten, die auch Informationen zu den rechtlichen Rahmenbedingungen enthalten, lassen sich Interessierte deutlich besser ansprechen, damit zukünftig

58 Vgl. Kulzer (2015) $195 f$. 
noch weitere engagierte Bürger^innen den Weg ins Ehrenamt bei Bibliotheken finden.

\begin{tabular}{|c|c|}
\hline \multicolumn{2}{|c|}{ Rechtliche Checkliste für Tätigkeitsvereinbarungen mit Ehrenamtlichen } \\
\hline Im Vorweg zu klären & Liegen Sonderbestimmungen vor? BFD, FSJ oder Pflichtpraktika \\
\hline & Wer ist Vertragspartner? \\
\hline \multirow[t]{2}{*}{ Vertragsschluss } & Form- und Inhaltsfreiheit \\
\hline & jederzeit beidseitig kündbar \\
\hline \multirow[t]{6}{*}{ Vertraglich zu regeln } & Inhalt der Dienstleistung \\
\hline & Ehrenamtlichkeit und Unentgeltlichkeit der Tätigkeit \\
\hline & Weisungsrecht der Bibliothek \\
\hline & Aufwandsentschädigung \\
\hline & Informationspflichten \\
\hline & Geheimnisschutz \\
\hline Datenschutzverpflichtung & Unterschrift erforderlich \\
\hline Verpflichtungserklärung & Unterschrift erforderlich \\
\hline Informieren / Hinweisen & Unfallversicherung/ Haftpflicht/ Steuerrecht \\
\hline \multirow[t]{2}{*}{ Dokumentation } & Tätigkeitsvereinbarung, Datenschutz-/Verpflichtungserklärung \\
\hline & Personalien und Führungszeugnis \\
\hline
\end{tabular}

Abb. 2: Checkliste hinsichtlich rechtlicher Anforderungen, die beim Abschluss einer Tätigkeitsvereinbarung mit Ehrenamtlichen zu beachten sind

\section{Literaturverzeichnis}

Gödan, Jürgen Christoph (1999): Mustervertrag für ehrenamtlich Tätige in Bibliotheken: Mit Erläuterungen. In: Bibliotheksdienst, 33 (6), 987-1000. Verfügbar unter https://www.bibliotheksver band.de/fileadmin/user_upload/Kommissionen/Kom_Recht/P ublikationen_Organisation/1999_Goedan_Mustervertrag_ehren amtlich.pdf.

Keite, Uta (2019): Professionell organisiertes Ehrenamt als integraler Bestandteil der Bibliotheksarbeit: Zukunftsweisendes Bürgerengagement am Beispiel der Bücherhallen Hamburg. In: Öffentliche Bibliothek 2030. Herausforderungen - Konzepte - Visionen, hg. v. Petra Hauke, 125-34. Bad Honnef: Bock + Herchen Verlag. Verfügbar unter https://doi.org/10.18452/20204.

Kulzer, (2015): Ehrenamt in Bibliotheken - Erfolg durch professionelles Management. In: Bibliotheksforum Bayern, (9), 194-96. Verfügbar unter https://www.bibliotheksforum-bayern.de/fi leadmin/archiv/2015-3/PDF-Einzelbeitraege/BFB_0315_09_Kul zer_V04.pdf.
Neumayer, Christoph (2001): Die Vertretung öffentlich-rechtlicher Körperschaften. In: Rheinische Notar-Zeitschrift, (6), 249-69.

Säcker, Franz Jürgen (Hrsg.) (2020): Münchener Kommentar zum Bürgerlichen Gesetzbuch. Bd. 6. 8. Aufl. München: Beck.

Umlauf, Konrad (2011): Management Bürgerschaftlichen Engagements in Bibliotheken. In: Erfolgreiches Management von Bibliotheken und Informationseinrichtungen: Fachratgeber für die Bibliotheksleitung und Bibliothekare, hg. v. Hans-Christoph Hobohm und Konrad Umlauf, Loseblattsammlung, Stand Juni 2020, Kap. 4.7. Hamburg: Dashöfer.

Zeddies, Ruth (2011a): Bürgerschaftliches Engagement in Bibliotheken: ein Handbuch für das Ehrenamts-Management. Hg. v. Deutschen Bibliotheksverband. Verfügbar unter https://www.biblio theksverband.de/fileadmin/user_upload/DBV/themen/ehren amt/Bürgerschaftliches_Engagment_in_Bibliotheken_Hand buch_2011.pdf.

Zeddies, Ruth (2011b): Das Ehrenamt in öffentlichen Bibliotheken kirchlicher Trägerschaft. Verfügbar unter https://www.biblio theksverband.de/fileadmin/user_upload/DBV/themen/ ehrenamt/Zeddies_Das_Ehrenamt_in_\%C3\%96ffentlichen_ Bibliotheken_kirchlicher_Tr\%C3\%A4gerschaft.pdf.

Zilkens, Martin (2019): Allgemeine Schutzbestimmungen. In: Datenschutz in der Kommunalverwaltung: Recht - Technik - Organisation, hg. v. Martin Zilkens und Lutz Gollan, 91-104. 5. Aufl. Berlin: Erich Schmidt Verlag.

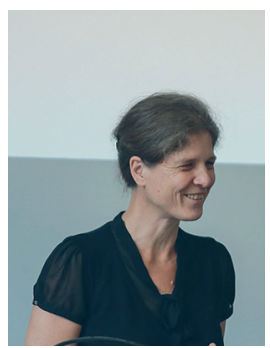

\section{Prof. Dr. Ulrike Verch}

Hochschule für Angewandte

Wissenschaften Hamburg

Institut für Medienrecht Hamburg

Finkenau 35

D-22081 Hamburg

ulrike.verch@haw-hamburg.de 